\title{
Modified Variational Iteration Method for Solving Nonlinear Partial Differential Equation Using Adomian Polynomials
}

\author{
S. A. Ojobor ${ }^{*}$, A. Obihia \\ Department of Mathematics, Faculty of Science, Delta State University, Abraka, Nigeria
}

Received March 18, 2021; Revised May 16, 2021; Accepted June 6, 2021

\section{Cite This Paper in the following Citation Styles}

(a): [1] S. A. Ojobor, A. Obihia , "Modified Variational Iteration Method for Solving Nonlinear Partial Differential Equation Using Adomian Polynomials," Mathematics and Statistics, Vol. 9, No. 4, pp. 456 - 464, 2021. DOI: 10.13189/ms.2021.090406.

(b): S. A. Ojobor, A. Obihia (2021). Modified Variational Iteration Method for Solving Nonlinear Partial Differential Equation Using Adomian Polynomials. Mathematics and Statistics, 9(4), 456 - 464. DOI: 10.13189/ms.2021.090406.

Copyright $(2021$ by authors, all rights reserved. Authors agree that this article remains permanently open access under the terms of the Creative Commons Attribution License 4.0 International License

\begin{abstract}
The aim of this paper is to solve numerically the Cauchy problems of nonlinear partial differential equation (PDE) in a modified variational iteration approach. The standard variational iteration method (VIM) is first studied before modifying it using the standard Adomian polynomials in decomposing the nonlinear terms of the PDE to attain the new iterative scheme modified variational iteration method (MVIM). The VIM was used to iteratively determine the nonlinear parabolic partial differential equation to obtain some results. Also, the modified VIM was used to solve the nonlinear PDEs with the aid of Maple 18 software. The results show that the new scheme MVIM encourages rapid convergence for the problem under consideration. From the results, it is observed that for the values the MVIM converges faster to exact result than the VIM though both of them attained a maximum error of order $10^{-9}$. The resulting numerical evidences were competing with the standard VIM as to the convergence, accuracy and effectiveness. The results obtained show that the modified VIM is a better approximant of the above nonlinear equation than the traditional VIM. On the basis of the analysis and computation we strongly advocate that the modified with finite Adomian polynomials as decomposer of nonlinear terms in partial differential equations and any other mathematical equation be encouraged as a numerical method.
\end{abstract}

Keywords Variational Iteration Method-VIM,
Adomian Polynomials, Cauchy Problems, Partial Differential Equation-PDE

\section{Introduction}

Real life situations are often modeled using partial differential equations (PDEs) because they possess the attribute of expressing more than one variable. Popular partial differential equations that have physical significant include: $u_{x x}-1 /{ }_{c^{2}} u_{t t}=0$. This equation has a magnificent application in the area of signal processing. Similarly, the diffusion equation (otherwise called the heat equation): $u_{x x}+u_{y y}=u_{t}$, describes the temperature distribution in a two-dimensional region in space and time. Also, it is significant in the study of reaction-diffusion systems such as the convection-reaction-diffusion systems; the Poission equation: $u_{x x}+u_{y y}+u_{z z}=g(x, y, z)$, is a very essential equation of mathematical physics that studies the spatial variation of potential function for given non-homogeneous term. It has a wide range of real-life applications in the modeling of ocean and electrostatics; the Navier Stokes equations [2]: $\partial \vec{u} / \partial t+(\vec{u} . \Delta) \vec{u}+$ $1 / \tau \Delta \tau-\gamma \Delta^{2} \vec{u}=\frac{1}{\tau} \vec{F}$. This equation is most relevant in computational fluid dynamics, establishing a superb relationship between external forces and pressure acting on a fluid in the direction of the fluid flow. Also, various 
forms of this equation are applicable in computations for ship designs, climate modeling, aircraft, weather forecasting, etc.; the Schrödodinger equation (Adebiyi and Fatumu, $\quad$ 2006): $\quad h^{2} / 8 \pi^{2} \Delta^{2} \psi(r, t)+V \psi(r, t)+$ $h / 2 \pi i \quad \partial \psi(r, t) / \partial t=0$ is the primary equation of quantum mechanics. When expressed in time-dependent form, it is widely used in the study of chemical reactions and in the development of chemical weapons.

Over the years, researchers have come up with relevant mathematical algorithms to explore and solve partial differential equations due to their wide range of applications to real-life situations. So far, researchers have been able to come up with methods which can be classified as either analytic or numerical methods. The analytic methods such as the d-expansion method, change of variable method, separation of variable method, etc., are really freaky, complex and difficult to execute requiring either linearization, quasi-linearization, perturbation, large computational effort, etc., Also, computational errors and round-off errors are very much renowned in the analytic methods which offers inconsistent interpretation in question with no regard to the internal and external characteristics of the model. To this effect, numerical methods (otherwise known as approximation methods) have become more suitable methods for linear and nonlinear PDE's. This is so because, numerical methods offer an approximate series solution of the analytic solution of the model in question. They are the direct opposite of the analytics methods requiring no hidden transformation, or any form of linearization. They are programmable and efficient. Popular numerical approaches include, the finite element method (FEM), the finite difference method (FDM), the Crank-Nicolsone scheme (CNS), the Bender-Scmidth method (BSM), the VIM, the ADM ([3] [17]) etc.

Recently, some researchers generalized the nonlinear evolution equations (NLEEs) to new one and obtain lump solution ([18], [19]).

The Chinese mathematician, He [7] proposed the variational iteration method (VIM). The method over the years has gained popularity with wide range applications to many areas of mathematics such as; integral equations, boundary and initial value problems, integro-differential problems, delay differential equations, stochastic differential equations, problems involving partial derivatives, etc.

Now, given the differential equation be given

$$
\mathrm{L}\{\mathrm{u}(\mathrm{x}, \mathrm{t})\}+\mathrm{R}\{\mathrm{u}(\mathrm{x}, \mathrm{t})\}+\mathrm{N}\{\mathrm{u}(\mathrm{x}, \mathrm{t})\}=\mathrm{g}(\mathrm{x})
$$

with some prescribed conditions, $u(x, t)$ is the unknown function, $\mathrm{L}$ is linear differential operator of the highest order, $\mathrm{R}$ is also a linear differential operator of order less than $\mathrm{L}, \mathrm{N}$ is nonlinear term and $\mathrm{g}(\mathrm{x})$ is the source term.

The VIM involves the construction of a correction functional for (1) given as

$$
\begin{array}{r}
\mathrm{u}_{\mathrm{k}+1}(\mathrm{x}, \mathrm{t})=\mathrm{u}_{\mathrm{k}}(\mathrm{x}, \mathrm{t})+\int_{0}^{\mathrm{x}} \lambda(\mathrm{x}, \mathrm{s})\left[\mathrm{L}\left\{\mathrm{u}_{\mathrm{k}}(\mathrm{x}, \mathrm{s})\right\}+\right. \\
\left.\mathrm{R}\left\{\mathrm{u}_{\mathrm{k}}(\mathrm{x}, \mathrm{s})\right\}+\mathrm{N}\left\{\tilde{\mathrm{u}}_{\mathrm{k}}(\mathrm{x}, \mathrm{s})\right\}-\mathrm{g}(\mathrm{x})\right] \mathrm{ds},(2)
\end{array}
$$

where $\mathrm{k}$ is a positive integer greater than zero, $\lambda(\mathrm{x}, \mathrm{s})$ is called the general language multiplier obtained optimally via the variational theorem, and $\tilde{\mathrm{u}}_{\mathrm{k}}(\mathrm{x}, \mathrm{s})=0$, called the restricted variable. The work of [9] gives the general language multiplier, $\lambda(\mathrm{x}, \mathrm{s})$ as

$$
\lambda(\mathrm{x}, \mathrm{s})=(-1)^{\mathrm{n}} \frac{(\mathrm{s}-\mathrm{x})^{(\mathrm{n}-1)}}{(\mathrm{n}-1) !},
$$

wheren denotes order of the given differential equation.

The plan of this article is to decipher numerically the Cauchy problems of nonlinear PDE of the form:

$$
\left(\frac{\partial \mathrm{u}}{\partial \mathrm{t}}-\Delta\right)\left(\frac{\partial^{2} \mathrm{u}}{\partial \mathrm{t}^{2}}-\Delta\right)-\mathrm{F}(\mathrm{u})=0,
$$

where $\mathrm{F}(\mathrm{u})$ is the nonlinear term, and $\Delta$ is a Laplace operator defined in $\mathbb{R}^{1}$. Specifically, equation (4) is a special class of the hyperbolic-parabolic PDE. For this purpose, we employ a modified version of VIM using Adomian polynomials in decomposing the nonlinear terms of the PDE. Maple 18 software is used implementing all the computations in this research.

\section{Standard Variational Iteration Method}

Using equation (2), VIM for (4) becomes:

$$
\begin{array}{r}
\mathrm{u}_{\mathrm{k}+1}(\mathrm{x}, \mathrm{t})=\mathrm{u}_{\mathrm{k}}(\mathrm{x}, \mathrm{t})+\int_{0}^{\mathrm{t}} \lambda(\mathrm{x}, \xi)\left[( ( \mathrm { u } _ { \mathrm { k } } ) _ { \xi } - \Delta ) \left(\left(\mathrm{u}_{\mathrm{k}}\right)_{\xi \xi}-\right.\right. \\
\left.\Delta)-\mathrm{Fu}_{\mathrm{k}}\right] \mathrm{d} \xi, \mathrm{k} \geq 0
\end{array}
$$

For convenience sake, we can rewrite (5) as:

$$
\begin{array}{r}
\mathrm{u}_{\mathrm{k}+1}(\mathrm{x}, \mathrm{t})=\mathrm{u}_{\mathrm{k}}(\mathrm{x}, \mathrm{t})+\int_{0}^{\mathrm{t}} \lambda(\mathrm{x}, \xi)\left[( \frac { \partial } { \partial \xi } - \Delta ) \left(\frac{\partial^{2}}{\partial \xi^{2}}-\right.\right. \\
\left.\Delta) \mathrm{u}_{\mathrm{k}}(\mathrm{x}, \xi)-\mathrm{F}\left(\mathrm{u}_{\mathrm{k}}(\mathrm{x}, \xi)\right)\right] \mathrm{d} \xi \quad(6)
\end{array}
$$

Equation (6) can be transformed to the form;

$$
\begin{array}{r}
u_{k+1}(x, t)=u_{k}(x, t)+\int_{0}^{t} \lambda(x, \xi)\left[u_{k}(\xi)-\right. \\
\left.k_{1} u_{k}\right] d \xi,(7)
\end{array}
$$

where

$$
\mathrm{u}_{\mathrm{k}}(\xi) \equiv\left(\frac{\partial}{\partial \xi}-\Delta\right)\left(\frac{\partial^{2}}{\partial \xi^{2}}-\Delta\right)=\mathrm{u}_{\mathrm{k}}(\xi) \text { and } \mathrm{G}\left(\mathrm{u}_{\mathrm{k}}(\mathrm{x}, \xi)\right) \equiv
$$

Thus, taking the variation $\delta$ on both sides of (7) to get;

$$
\begin{gathered}
=\delta u_{\mathrm{t}}(\mathrm{x}, \mathrm{t}) \\
\left.+\delta \int_{0} \lambda(\mathrm{x}, \xi)\left[\mathrm{u}_{\mathrm{k}}(\xi)-\mathrm{k}_{1} \mathrm{u}_{\mathrm{k}}\right] \mathrm{x}, \mathrm{t}\right), \\
\Rightarrow \delta \mathrm{u}_{\mathrm{k}+1}(\mathrm{x}, \mathrm{t})=\delta \mathrm{u}_{\mathrm{k}}(\mathrm{x}, \mathrm{t})+\int_{0}^{\mathrm{t}} \lambda(\mathrm{x}, \xi) \delta \mathrm{u}_{\mathrm{k}}(\xi) \mathrm{d} \xi- \\
\int_{0}^{\mathrm{t}} \mathrm{k}_{1} \lambda(\mathrm{x}, \xi) \delta \mathrm{u}_{\mathrm{k}}(\mathrm{x}, \xi) \mathrm{d} \xi(8 \mathrm{a})
\end{gathered}
$$

By integration by part, the first integral gives 
Let $\mathrm{u}=\lambda(\mathrm{x}, \xi) \Rightarrow \mathrm{du}=\lambda^{\prime}(\mathrm{x}, \xi) \mathrm{d} \xi ; \mathrm{dv}=\delta\left(\mathrm{u}_{\mathrm{k}}\right)(\xi) \Rightarrow \mathrm{v}=$ $\delta u_{\mathrm{k}}(\mathrm{x}, \xi)$

$$
\begin{aligned}
\delta u_{k+1}(x, t) & =\delta u_{k}(x, t)+\left.\lambda(x, \xi) \delta u_{k}(\xi)\right|_{\xi=t} \\
& -\int_{0}^{t} \lambda^{\prime}(x, \xi) \delta u_{k}(\xi) d \xi \\
& -\int_{0}^{t} k_{1} \lambda(x, \xi) \delta u_{k}(x, \xi) d \xi
\end{aligned}
$$

Applying extremum condition, $\lambda^{\prime}(x, \xi)+\mathrm{k}_{1} \lambda(\mathrm{x}, \xi)=0$

Solving equation (8a) with initial condition $1+$ $\left.\lambda(\mathrm{x}, \xi)\right|_{\xi=\mathrm{t}}=0$, gives

$$
\lambda(\mathrm{x}, \xi)=-\mathrm{e}^{-\mathrm{k}_{1}(\xi-\mathrm{t})}, \mathrm{k}_{1} \neq 0 .
$$

Thus, the variational scheme for the nonlinear parabolic - hyperbolic Partial differential equation (4) is given as

$\mathrm{u}_{\mathrm{k}+1}(\mathrm{x}, \mathrm{t})=\mathrm{u}_{\mathrm{k}}(\mathrm{x}, \mathrm{t})-\int_{0}^{\mathrm{t}} \mathrm{e}^{-\mathrm{k}_{1}(\xi-\mathrm{t})}\left[\left(\left(\mathrm{u}_{\mathrm{k}}\right)_{\xi}-\Delta\right)\left(\left(\mathrm{u}_{\mathrm{k}}\right)_{\xi \xi}-\right.\right.$

$\left.\Delta)-\mathrm{Fu}_{\mathrm{k}}\right] \mathrm{d} \xi, \mathrm{k} \geq 0$ (10)

\section{Modified Variational Iteration Method-VIM}

This method takes the usual pattern of the VIM except that the nonlinear component in (4) is first decomposed using Adomian polynomials, that is,

$$
\begin{aligned}
& \mathrm{F}(\mathrm{u})=\sum_{\mathrm{r}=0}^{\mathrm{k}} \mathrm{A}_{\mathrm{r}}, \mathrm{k} \geq 0, \quad \text { Using the MVIM (13) with Maple } 18 \text { softw } \\
& u_{1}:=-\mathrm{e}^{x}-\mathrm{e}^{t+2 x}+\mathrm{e}^{2 x}+2 \mathrm{e}^{t+x} \\
& u_{2}:=\mathrm{e}^{x}+8 \mathrm{e}^{t+2 x}-3 \mathrm{e}^{2 x}-4 \mathrm{e}^{t+x}-9 \mathrm{e}^{3 t+4 x}-6 \mathrm{e}^{2 t+3 x}+33 \mathrm{e}^{2 t+4 x}+14 \mathrm{e}^{t+3 x} \\
&-40 \mathrm{e}^{t+4 x}-5 \mathrm{e}^{2 t+2 x}+4 \mathrm{e}^{2 t+x}-8 \mathrm{e}^{3 x}+16 \mathrm{e}^{4 x} \\
& u_{3}:=-\mathrm{e}^{x}-36864 \mathrm{e}^{7 x}+2240 \mathrm{e}^{5 x}+366864 \mathrm{e}^{3 t+7 x}-624 \mathrm{e}^{5 t+4 x}+8484 \mathrm{e}^{5 t+5 x} \\
&-202908 \mathrm{e}^{4 t+7 x}-1656 \mathrm{e}^{6 t+5 x}-630 \mathrm{e}^{6 t+6 x}+56808 \mathrm{e}^{5 t+7 x}-6156 \mathrm{e}^{6 t+7 x} \\
&+504 \mathrm{e}^{4 t+3 x}+11066 \mathrm{e}^{2 t+5 x}-13400 \mathrm{e}^{4 t+5 x}+180608 \mathrm{e}^{t+7 x}-9584 \mathrm{e}^{t+5 x} \\
&+2850 \mathrm{e}^{3 t+5 x}-120 \mathrm{e}^{5 t+3 x}-358352 \mathrm{e}^{2 t+7 x}-372736 \mathrm{e}^{t+8 x}+65536 \mathrm{e}^{8 x} \\
&+11520 \mathrm{e}^{t+6 x}-960 \mathrm{e}^{6 x}-48 \mathrm{e}^{5 t+2 x}+48 \mathrm{e}^{4 t+2 x}+886848 \mathrm{e}^{2 t+8 x} \\
&-1140528 \mathrm{e}^{3 t+8 x}+44616 \mathrm{e}^{3 t+6 x}-34488 \mathrm{e}^{2 t+6 x}-366390 \mathrm{e}^{5 t+8 x} \\
&+851832 \mathrm{e}^{4 t+8 x}+7650 \mathrm{e}^{5 t+6 x}-27708 \mathrm{e}^{4 t+6 x}-7371 \mathrm{e}^{7 t+8 x}+82809 \mathrm{e}^{6 t+8 x} \\
&+16 \mathrm{e}^{3 t+x}-19 \mathrm{e}^{3 t+2 x}-638 \mathrm{e}^{3 t+3 x}+2449 \mathrm{e}^{4 t+4 x}+48 \mathrm{e}^{3 x}-256 \mathrm{e}^{4 x} \\
&+54 \mathrm{e}^{2 t+2 x}-20 \mathrm{e}^{2 t+x}+560 \mathrm{e}^{t+4 x}+854 \mathrm{e}^{2 t+4 x}-134 \mathrm{e}^{t+3 x}-2983 \mathrm{e}^{3 t+4 x} \\
&+340 \mathrm{e}^{2 t+3 x}+13 \mathrm{e}^{2 x}-48 \mathrm{e}^{t+2 x}+6 \mathrm{e}^{t+x}
\end{aligned}
$$

where $A_{r}, r \geq 0$ are called Adomian polynomials which generate recursively using

$$
\frac{1}{\mathrm{k} !} \frac{\mathrm{d}^{\mathrm{k}}}{\mathrm{d} \lambda^{\mathrm{k}}}\left[\mathrm{N}\left(\mathrm{t}, \sum_{\mathrm{j}=0}^{\infty} \lambda^{\mathrm{i}} \mathrm{u}_{\mathrm{i}}(\mathrm{t}), \sum_{\mathrm{j}=0}^{\infty} \lambda^{\mathrm{i}} \mathrm{u}_{\mathrm{i}}(\mathrm{u}(\mathrm{x}, \mathrm{t}))\right]_{\lambda=0}\right.
$$

Substituting (11) into the iterative scheme (10), we have

$$
\begin{array}{r}
\mathrm{u}_{\mathrm{k}+1}(\mathrm{x}, \mathrm{t})=\mathrm{u}_{\mathrm{k}}(\mathrm{x}, \mathrm{t})-\int_{0}^{\mathrm{t}} \mathrm{e}^{-\mathrm{k}_{1}(\xi-\mathrm{t})}\left[( ( \mathrm { u } _ { \mathrm { k } } ) _ { \xi } - \Delta ) \left(\left(\mathrm{u}_{\mathrm{k}}\right)_{\xi \xi}-\right.\right. \\
\left.\Delta)-\sum_{\mathrm{r}=0}^{\mathrm{k}} \mathrm{A}_{\mathrm{r}}(\mathrm{x}, \xi)\right] \mathrm{d} \xi \quad \mathrm{k} \geq 0,(13)
\end{array}
$$

Thus, the iteration scheme is called the MVIM. This treatment is for (4).

\section{Numerical Experiments}

Here, we apply the MVIM to nonlinear hyperbolic-parabolic PDEs as follows:

\section{Problem 1[1]:}

Given

$$
\left(\frac{\partial \mathrm{u}}{\partial \mathrm{t}}-\frac{\partial^{2} \mathrm{u}}{\partial \mathrm{x}^{2}}\right)\left(\frac{\partial^{2} \mathrm{u}}{\partial \mathrm{t}^{2}}-\frac{\partial^{2} \mathrm{u}}{\partial \mathrm{x}^{2}}\right)=\frac{\partial^{2} \mathrm{u}}{\partial \mathrm{x}^{2}}+\frac{\partial^{2} \mathrm{u}}{\partial \mathrm{t}^{2}}-\frac{\partial \mathrm{u}}{\partial \mathrm{t}}+\mathrm{u},
$$

with initial conditions

$$
\mathrm{u}(\mathrm{x}, 0)=\mathrm{e}^{\mathrm{x}},
$$

and analytic solution given as $\mathrm{u}(\mathrm{x}, \mathrm{t})=\mathrm{e}^{\mathrm{x}+\mathrm{t}}$.

Using the MVIM (13) with Maple 18 software, we have: 


\section{Problem 2 [1]:}

Consider

$$
\left(\frac{\partial \mathrm{u}}{\partial \mathrm{t}}-\frac{\partial^{2} \mathrm{u}}{\partial \mathrm{x}^{2}}\right)\left(\frac{\partial^{2} \mathrm{u}}{\partial \mathrm{t}^{2}}-\frac{\partial^{2} \mathrm{u}}{\partial \mathrm{x}^{2}}\right)=\frac{\partial^{4} \mathrm{u}}{\partial \mathrm{t}^{4}}+\frac{\partial^{2} \mathrm{u}}{\partial \mathrm{t}^{2}}-\mathrm{u} \frac{\partial \mathrm{u}}{\partial \mathrm{t}}+\frac{\partial^{2} \mathrm{u}}{\partial \mathrm{x}^{2}}-\frac{\partial \mathrm{u}}{\partial \mathrm{t}}+2 \mathrm{u}
$$

with initial condition $\mathrm{u}(\mathrm{x}, 0)=\cos \mathrm{x}$,

and exact solution given as $\mathrm{u}(\mathrm{x}, \mathrm{t})=\mathrm{e}^{2 \mathrm{t}} \cos \mathrm{x}$.

By MVIM , we have,

$$
\begin{aligned}
& u_{1}:=\frac{5}{2} \cos (x)-\frac{1}{2} \mathrm{e}^{2 t} \cos (x)^{2}-\frac{3}{2} \mathrm{e}^{2 t} \cos (x)+\frac{1}{2} \cos (x)^{2} \\
& u_{2}:= \frac{5}{2} \mathrm{e}^{6 t} \cos (x)^{2} \sin (x)^{2}+6 \mathrm{e}^{6 t} \cos (x) \sin (x)^{2}-\frac{3}{4} \mathrm{e}^{4 t} \cos (x)^{2} \sin (x) \\
&-\frac{9}{4} \mathrm{e}^{4 t} \cos (x) \sin (x)-6 \mathrm{e}^{4 t} \cos (x)^{2} \sin (x)^{2}-\frac{29}{2} \mathrm{e}^{4 t} \cos (x) \sin (x)^{2} \\
&-\frac{149}{8} \mathrm{e}^{2 t} \cos (x)^{2}-3 \mathrm{e}^{2 t} \cos (x)+4 \cos (x)-3 \mathrm{e}^{2 t} \cos (x)^{4}-\frac{3}{2} \mathrm{e}^{2 t} \sin (x)^{4} \\
&-\frac{59}{4} \mathrm{e}^{2 t} \cos (x)^{3}-\sin (x)^{2} \cos (x)^{2}-\frac{3}{4} \sin (x) \cos (x)^{2}-\frac{5}{2} \cos (x) \sin (x)^{2} \\
&-\frac{15}{4} \sin ^{2}(x) \cos (x)+\frac{9}{2} \mathrm{e}^{2 t} \cos (x)^{2} \sin (x)^{2}+\frac{3}{2} \sin (x) \mathrm{e}^{2 t} \cos (x)^{2} \\
&+11 \mathrm{e}^{2 t} \cos (x) \sin (x)^{2}+6 \sin (x) \mathrm{e}^{2 t} \cos (x)-3 \mathrm{e}^{6 t} \cos (x)^{4}-\frac{1}{2} \mathrm{e}^{6 t} \sin (x)^{4} \\
&-\frac{57}{4} \mathrm{e}^{6 t} \cos (x)^{3}-\frac{135}{8} \mathrm{e}^{6 t} \cos (x)^{2}+\frac{255}{8} \mathrm{e}^{4 t} \cos (x)^{2}+\frac{11}{2} \mathrm{e}^{4 t} \cos (x)^{4} \\
&+\frac{3}{2} \mathrm{e}^{4 t} \sin (x)^{4}+\frac{53}{2} \mathrm{e}^{4 t} \cos (x)^{3}+\frac{29}{8} \cos (x)^{2}+\frac{1}{2} \cos (x)^{4}+\frac{1}{2} \sin (x)^{4} \\
&+\frac{5}{2} \cos (x)^{3}
\end{aligned}
$$

\section{Problem 3 [1]:}

$$
\left(\frac{\partial \mathrm{u}}{\partial \mathrm{t}}-\frac{\partial^{2} \mathrm{u}}{\partial \mathrm{x}_{1}{ }^{2}}-\frac{\partial^{2} \mathrm{u}}{\partial \mathrm{x}_{2}{ }^{2}}\right)\left(\frac{\partial^{2} \mathrm{u}}{\partial \mathrm{t}^{2}}-\frac{\partial^{2} \mathrm{u}}{\partial \mathrm{x}_{1}{ }^{2}}-\frac{\partial^{2} \mathrm{u}}{\partial \mathrm{x}_{2}{ }^{2}}\right)=\frac{\partial \mathrm{u}}{\partial \mathrm{x}_{1}}-\frac{\partial^{2} \mathrm{u}}{\partial \mathrm{t}^{2}}+\mathrm{u}
$$

with initial conditions $u\left(x_{1}, x_{2}, 0\right)=e^{x_{1}+x_{2}}$,

The exact solution is given as $\mathrm{u}(\mathrm{x}, \mathrm{t})=\mathrm{e}^{\mathrm{x}_{1}+\mathrm{x}_{2}+\mathrm{t}}$.

By MVIM , we have,

$$
\begin{aligned}
u_{1}:=\mathrm{e}^{x_{1}+x_{2}}-4 \mathrm{e}^{t+2 x_{1}+2 x_{2}}+4 \mathrm{e}^{2 x_{1}+2 x_{2}} & \\
u_{2}:= & \mathrm{e}^{x_{1}+x_{2}}-8 \mathrm{e}^{t+2 x_{1}+2 x_{2}}+8 \mathrm{e}^{2 x_{1}+2 x_{2}}-784 \mathrm{e}^{3 t+4 x_{1}+4 x_{2}}+112 \mathrm{e}^{2 t+3 x_{1}+3 x_{2}} \\
& +2576 \mathrm{e}^{2 t+4 x_{1}+4 x_{2}}-240 \mathrm{e}^{t+3 x_{1}+3 x_{2}}-2816 \mathrm{e}^{t+4 x_{1}+4 x_{2}}+128 \mathrm{e}^{3 x_{1}+3 x_{2}} \\
& +1024 \mathrm{e}^{4 x_{1}+4 x_{2}}
\end{aligned}
$$




$$
\begin{aligned}
& u_{3}:=\mathrm{e}^{x_{1}+x_{2}}+150994944 \mathrm{e}^{7 x_{1}+7 x_{2}}+425984 \mathrm{e}^{5 x_{1}+5 x_{2}}-409975552 \mathrm{e}^{7 t+8 x_{1}+8 x_{2}} \\
& -6794772480 \mathrm{e}^{t+8 x_{1}+8 x_{2}}-820641792 \mathrm{e}^{t+7 x_{1}+7 x_{2}}+269696 \mathrm{e}^{4 t+5 x_{1}+5 x_{2}} \\
& -5092864 \mathrm{e}^{5 t+6 x_{1}+6 x_{2}}+67963392 \mathrm{e}^{6 t+7 x_{1}+7 x_{2}}-12166350336 \mathrm{e}^{5 t+8 x_{1}+8 x_{2}} \\
& -1240512 \mathrm{e}^{3 t+5 x_{1}+5 x_{2}}-484725248 \mathrm{e}^{5 t+7 x_{1}+7 x_{2}}+2100288 \mathrm{e}^{2 t+5 x_{1}+5 x_{2}} \\
& -1555456 \mathrm{e}^{t+5 x_{1}+5 x_{2}}-68411904 \mathrm{e}^{3 t+6 x_{1}+6 x_{2}}+1413590528 \mathrm{e}^{4 t+7 x_{1}+7 x_{2}} \\
& \begin{array}{l}
+1836318720 \mathrm{e}^{2 t+7 x_{1}+7 x_{2}}-43147264 \mathrm{e}^{t+6 x_{1}+6 x_{2}}-2163500544 \mathrm{e}^{3 t+7 x_{1}+7 x_{2}} \\
-26894999552 \mathrm{e}^{3 t+8 x_{1}+8 x_{2}}+29777664 \mathrm{e}^{4 t+6 x_{1}+6 x_{2}}+3443390720 \mathrm{e}^{6 t+8 x_{1}+8 x_{2}}
\end{array} \\
& -26894999552 \mathrm{e}^{2}+29777664 \mathrm{e} \quad 1-2443390720 \mathrm{e} \\
& +23511541760 \mathrm{e}^{4 t+8 x_{1}+8 x_{2}}+18237423616 \mathrm{e}^{2 t+8 x_{1}+8 x_{2}} \\
& +77371648 \mathrm{e}^{2 t+6 x_{1}+6 x_{2}}+9502720 \mathrm{e}^{6 x_{1}+6 x_{2}}+1073741824 \mathrm{e}^{8 x_{1}+8 x_{2}} \\
& +14336 \mathrm{e}^{4 x_{1}+4 x_{2}}+384 \mathrm{e}^{3 x_{1}+3 x_{2}}-720 \mathrm{e}^{t+3 x_{1}+3 x_{2}}-39616 \mathrm{e}^{t+4 x_{1}+4 x_{2}} \\
& +35920 \mathrm{e}^{2 t+4 x_{1}+4 x_{2}}-10640 \mathrm{e}^{3 t+4 x_{1}+4 x_{2}}+336 \mathrm{e}^{2 t+3 x_{1}+3 x_{2}}+12 \mathrm{e}^{2 x_{1}+2 x_{2}} \\
& -12 \mathrm{e}^{t+2 x_{1}+2 x_{2}}
\end{aligned}
$$

\section{Problem 4. [1]:}

$$
\left(\frac{\partial \mathrm{u}}{\partial \mathrm{t}}-\frac{\partial^{2} \mathrm{u}}{\partial \mathrm{x}_{1}{ }^{2}}-\frac{\partial^{2} \mathrm{u}}{\partial \mathrm{x}_{2}{ }^{2}}\right)\left(\frac{\partial^{2} \mathrm{u}}{\partial \mathrm{t}^{2}}-\frac{\partial^{2} \mathrm{u}}{\partial \mathrm{x}_{1}{ }^{2}}-\frac{\partial^{2} \mathrm{u}}{\partial \mathrm{x}_{2}{ }^{2}}\right)=-3 \frac{\partial^{2} \mathrm{u}}{\partial \mathrm{x}_{2}{ }^{2}}-\frac{\partial^{2} \mathrm{u}}{\partial \mathrm{x}_{1}{ }^{2}}+\frac{5}{2} \frac{\partial^{2} \mathrm{u}}{\partial \mathrm{t}^{2}}+\frac{\partial \mathrm{u}}{\partial \mathrm{t}}+2 \mathrm{u}
$$

with initial conditions $\mathrm{u}\left(\mathrm{x}_{1}, \mathrm{x}_{2}, 0\right)=\sinh \mathrm{x}_{1}+\mathrm{e}^{\mathrm{x}_{2}}$, and exact solution given as $u\left(x_{1}, x_{2}, t\right)=\sinh x_{1}+e^{x_{2}+2 t}$.

By MVIM , we have,

$$
\begin{aligned}
u_{1}:=\frac{3}{2} \mathrm{e}^{x_{2}}-\frac{1}{2} \mathrm{e}^{2 x_{2}+2 t}+\mathrm{e}^{2 t} \sinh x_{1}-\frac{1}{2} \mathrm{e}^{x_{2}+2 t}+\frac{1}{2} \mathrm{e}^{2 x_{2}} \\
u_{2}:=-6 \mathrm{e}^{2 t} \sinh x_{1}+\mathrm{e}^{4 t+4 x_{2}}+\frac{9}{4} \mathrm{e}^{x_{2}}+\frac{3}{4} \mathrm{e}^{6 t+3 x_{2}}+2 \mathrm{e}^{4 x_{2}}-\frac{9}{2} \mathrm{e}^{x_{2}+2 t} \sinh x_{1} \\
-6 \mathrm{e}^{2 x_{2}+2 t} \sinh x_{1}-4 \mathrm{e}^{6 t} \sinh ^{2} x_{1}^{2}+7 \mathrm{e}^{4 t} \sinh x_{1}-\frac{3}{8} \mathrm{e}^{2 x_{2}+6 t}+8 \mathrm{e}^{4 t+2 x_{2}} \sinh x_{1} \\
+4 \mathrm{e}^{4 t} \sinh ^{2} x_{1}^{2}+\frac{5}{2} \mathrm{e}^{6 t+x_{2}} \sinh x_{1}+2 \mathrm{e}^{4 t+x_{2}} \sinh x_{1}-2 \mathrm{e}^{2 x_{2}+6 t} \sinh x_{1}+3 \mathrm{e}^{3 x_{2}} \\
-3 \mathrm{e}^{4 x_{2}+2 t}-\frac{7}{4} \mathrm{e}^{3 x_{2}+2 t}-2 \mathrm{e}^{3 x_{2}+4 t}-\frac{13}{8} \mathrm{e}^{4 t+2 x_{2}}-\frac{11}{4} \mathrm{e}^{4 t+x_{2}}+\frac{3}{2} \mathrm{e}^{x_{2}+2 t} \\
+\frac{33}{8} \mathrm{e}^{2 x_{2}}-\frac{17}{8} \mathrm{e}^{2 x_{2}+2 t}
\end{aligned}
$$




\section{Tables of Results}

Table 1. Comparison of results between exact solution, VIM [1] and MVIM for Problem 1

\begin{tabular}{|c|c|c|c|c|c|}
\hline$x$ & $\begin{array}{c}\text { Exact, } \\
u(x, t)\end{array}$ & $\begin{array}{c}V I M, \\
u_{3}(x, t)\end{array}$ & $\begin{array}{c}\text { VIM } \\
\text { error }\end{array}$ & $\begin{array}{c}\text { MVIM, } \\
u_{3}(x, t)\end{array}$ & $\begin{array}{c}\text { MVIM } \\
\text { error }\end{array}$ \\
\hline 0.00 & 1.0000000 & 1.0000500 & $5.0000 \mathrm{e}-05$ & 1.0001000 & $1.0000 \mathrm{e}-04$ \\
\hline 0.10 & 0.9950042 & 0.9950431 & $3.8962 \mathrm{e}-05$ & 0.9949066 & $9.7527 \mathrm{e}-05$ \\
\hline 0.20 & 0.9800666 & 0.9801026 & $3.5985 \mathrm{e}-05$ & 0.9799762 & $9.0423 \mathrm{e}-05$ \\
\hline 0.30 & 0.9553365 & 0.9553679 & $3.1458 \mathrm{e}-05$ & 0.9552569 & $7.9576 \mathrm{e}-06$ \\
\hline 0.40 & 0.9210610 & 0.9210869 & $2.5948 \mathrm{e}-05$ & 0.9209947 & $6.6289 \mathrm{e}-05$ \\
\hline 0.50 & 0.8775826 & 0.8776027 & $2.0095 \mathrm{e}-05$ & 0.8775305 & $5.2052 \mathrm{e}-07$ \\
\hline 0.60 & 0.8253356 & 0.8253501 & $1.4508 \mathrm{e}-05$ & 0.8252973 & $3.8296 \mathrm{e}-05$ \\
\hline 0.70 & 0.7648422 & 0.7648519 & $9.6646 \mathrm{e}-06$ & 0.7648160 & $2.6173 \mathrm{e}-07$ \\
\hline 0.80 & 0.6967067 & 0.6967126 & $5.8515 \mathrm{e}-06$ & 0.6966903 & $1.6415 \mathrm{e}-07$ \\
\hline 0.90 & 0.6216100 & 0.6216131 & $3.1474 \mathrm{e}-06$ & 0.6216007 & $9.2809 \mathrm{e}-06$ \\
\hline 1.00 & 0.5403023 & 0.5403038 & $1.4500 \mathrm{e}-06$ & 0.5402977 & $4.6045 \mathrm{e}-06$ \\
\hline
\end{tabular}

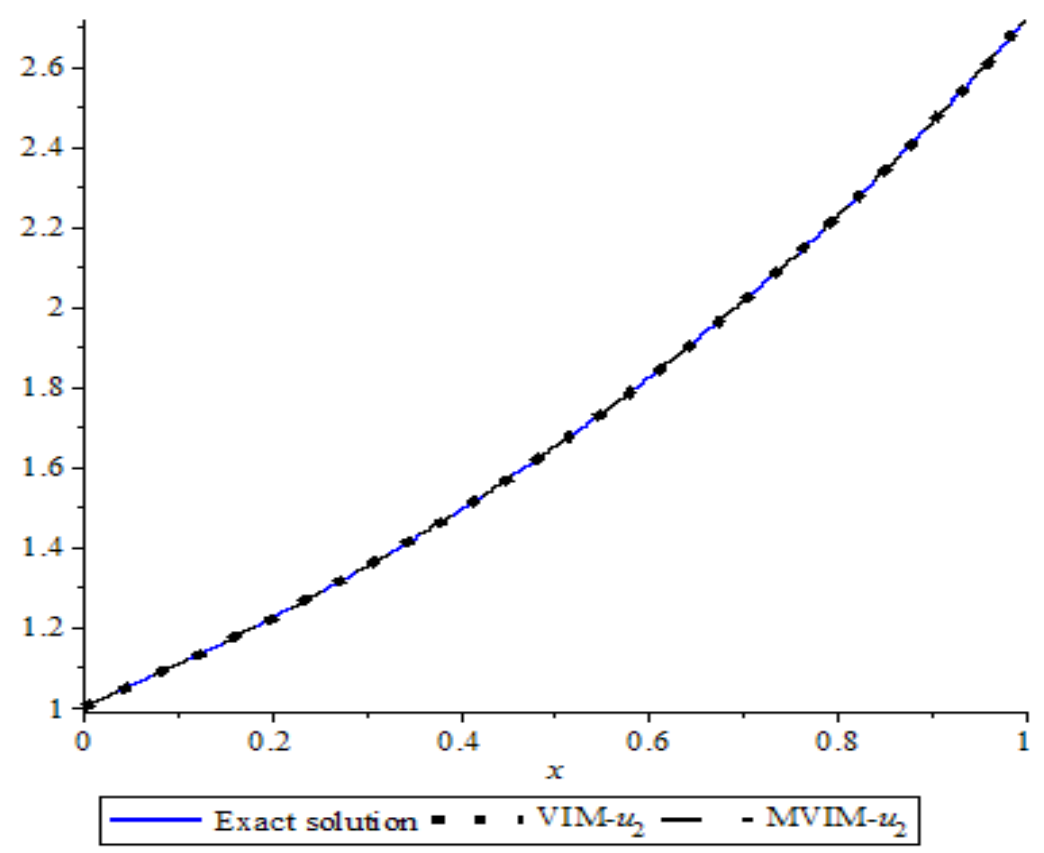

Figure 1. Graphical simulation the exact solution, VIM and MVIM for Problem 1

Table 2. Comparison of results between the exact solution, VIM [1] and MVIM Problem 2

\begin{tabular}{|c|c|c|c|c|c|}
\hline$x$ & $\begin{array}{c}\text { Exact, } \\
u(x, t)\end{array}$ & $\begin{array}{c}\text { VIM, } \\
u_{3}(x, t)\end{array}$ & $\begin{array}{c}\text { MVIM } \\
u_{2}(x, t)\end{array}$ & $\begin{array}{c}\text { VIM } \\
\text { error }\end{array}$ & $\begin{array}{c}\text { MVIM } \\
\text { error }\end{array}$ \\
\hline 0.00 & 1.0000000 & 1.0000000 & 1.0000000 & $0.0000 \mathrm{e}+00$ & $0.0000 \mathrm{e}+00$ \\
\hline 0.10 & 1.1051709 & 1.1051709 & 1.1051709 & $2.0000 \mathrm{e}-09$ & $2.0000 \mathrm{e}-09$ \\
\hline 0.20 & 1.2214028 & 1.2214028 & 1.2214028 & $2.0000 \mathrm{e}-09$ & $2.0000 \mathrm{e}-09$ \\
\hline 0.30 & 1.3498588 & 1.3498588 & 1.3498588 & $1.2000 \mathrm{e}-08$ & $7.2000 \mathrm{e}-08$ \\
\hline 0.40 & 1.4918247 & 1.4918248 & 1.4918247 & $6.2000 \mathrm{e}-08$ & $2.8000 \mathrm{e}-08$ \\
\hline 0.50 & 1.6487213 & 1.6487213 & 1.6487213 & $2.9000 \mathrm{e}-08$ & $1.2900 \mathrm{e}-07$ \\
\hline 0.60 & 1.8221188 & 1.8221188 & 1.8221188 & $0.0000 \mathrm{e}+00$ & $1.0000 \mathrm{e}-07$ \\
\hline 0.70 & 2.0137527 & 2.0137527 & 2.0137527 & $7.0000 \mathrm{e}-09$ & $7.0000 \mathrm{e}-09$ \\
\hline 0.80 & 2.2255409 & 2.2255409 & 2.2255409 & $2.8000 \mathrm{e}-08$ & $2.8000 \mathrm{e}-08$ \\
\hline 0.90 & 2.4596031 & 2.4596030 & 2.4596031 & $1.1100 \mathrm{e}-07$ & $1.1000 \mathrm{e}-08$ \\
\hline 1.00 & 2.7182818 & 2.7182824 & 2.7182819 & $5.7200 \mathrm{e}-07$ & $7.2000 \mathrm{e}-08$ \\
\hline
\end{tabular}




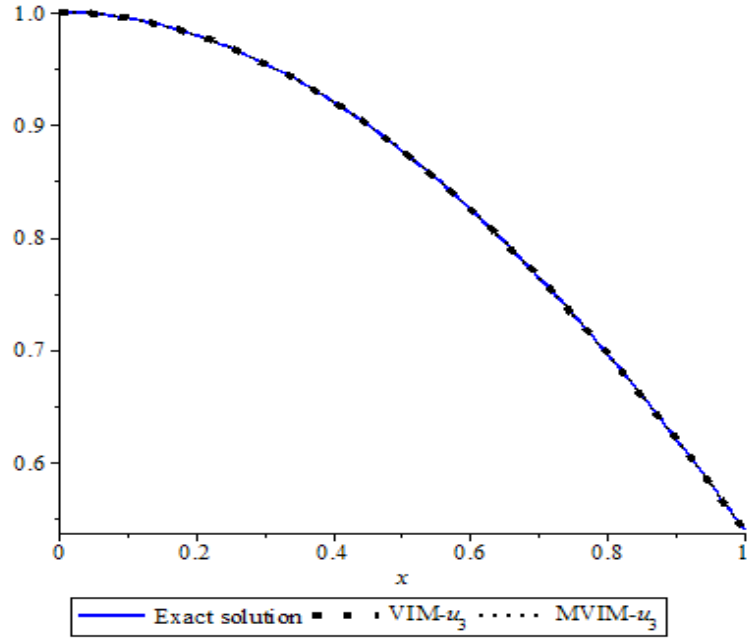

Figure 2. Graphical simulation of exact, VIM and MVIM for Problem 2

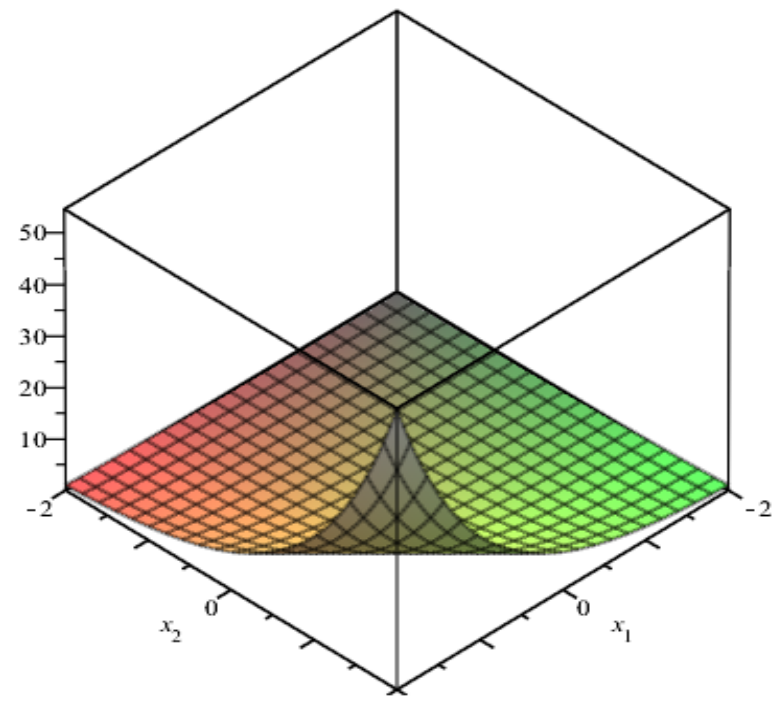

Figure 3a. Graphical simulation of exact for Problem 3

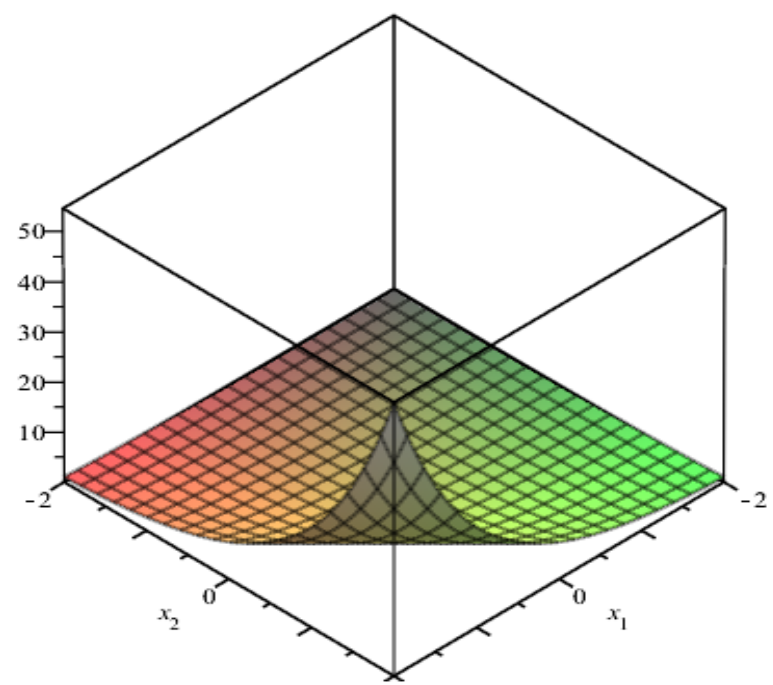

Figure 3b. Graphical simulation of estimated solution $\boldsymbol{u}_{3}$ for Problem 3 with VIM

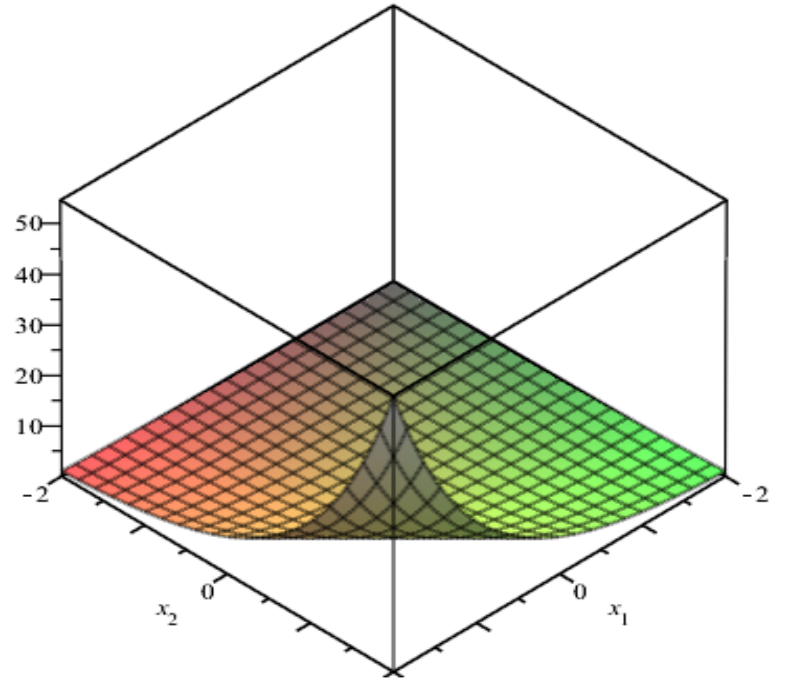

Figure 3c. Graphical simulation of estimated solution $\boldsymbol{u}_{\mathbf{3}}$ for Example 3 with MVIM

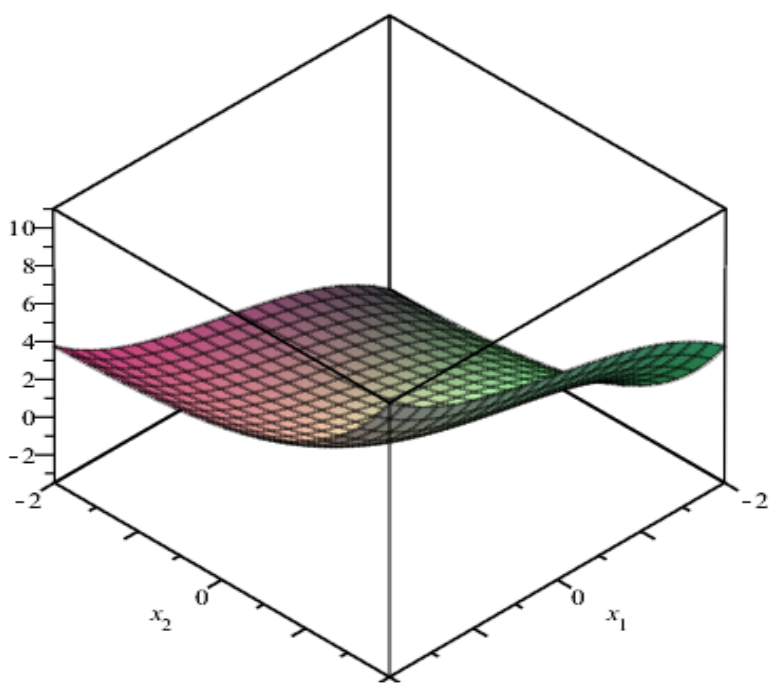

Figure 4a. Graphical simulation of exact solution for Problem 4

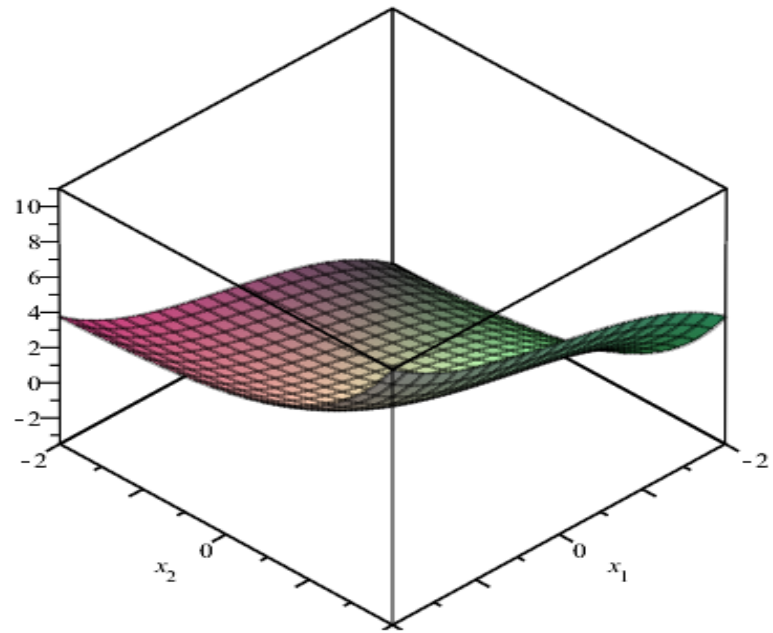

Figure 4b. Graphical simulation of approximate solution $\boldsymbol{u}_{3}$ for Problem 4 with VIM 


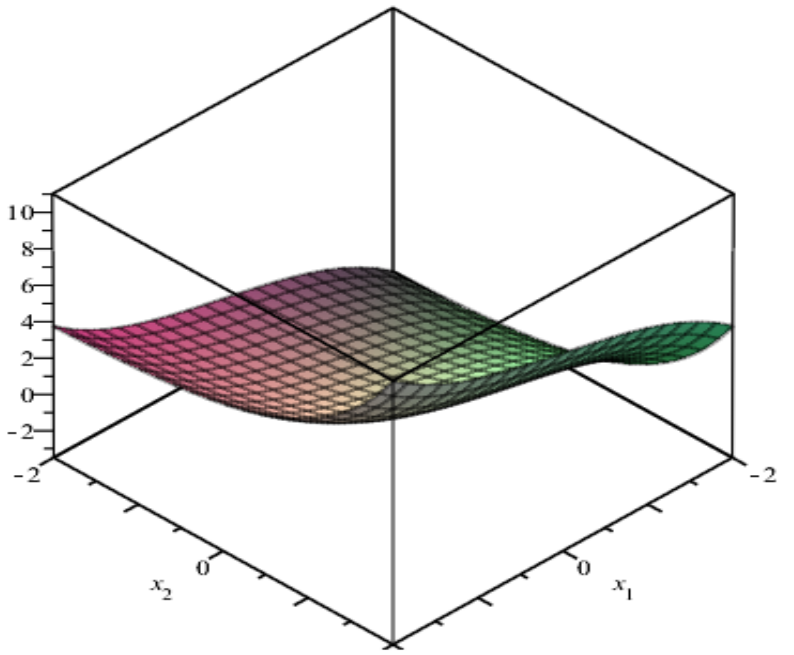

Figure 4c. Graphical simulation of approximate solution $\boldsymbol{u}_{3}$ for Example 4.4 with MVIM

\section{Discussion of Results}

We have successively applied the MVIM to the various forms of (4). Numerical evidences from MVIM were correlated with exact solution and VIM for accuracy and convergence. The following observations were captured.

In Problem 1, it is observed from table 1 and figure 1 that MVIM converges faster to exact solution than the VIM. Though both attained a minimum error of order $10^{-9}$, a careful observation at the various grid points in the table 1 vividly shows the superiority of MVIM over VIM. This is also evident in the graphical simulation of the problem as shown in figure 1.

In Problem 2, it is practical that MVIM attained a minimum error of order $10^{-7}$ against VIM with maximum error of order $10^{-6}$ as shown in Table 2 . This is so because the effects of decomposition of the nonlinear term by the Adomian polynomials were renowned in the iterative scheme. Thus, it is evident that MVIM converges better and more rapid to exact than the VIM as also seen in figure 2 .

In the Problems 3-4, we attained an absolute convergence at the initial value of $t$, this so because the effect of decomposition of nonlinear term by Adomian polynomials was absolute in the iterative scheme. Here, both the MVIM and VIM converges absolutely to exact solution as shown in the figures $3 a-3 b$ and $4 a-4 b$ respectively.

\section{Conclusions}

From the paper, we determined iteratively the Cauchy problem of nonlinear parabolic-hyperbolic PDE's of the form (3.1). We have been also to reformulate the standard VIM to obtain the new iterative scheme MVIM. We have been able to implement the new iterative scheme MVIM on some forms of Cauchy problem of nonlinear parabolic-hyperbolic PDE's. Also, comparison of results between the MVIM, VIM and exact solution were carried out, and are presented in tables and graphs. The results show MVIM converges better and faster to exact answer than the VIM.

\section{REFERENCES}

[1] S. Duangpithak, "Variational iteration method for special nonlinear partial differential equations," Int. Journal of Math. Analysis, vol. 6, no. 22, pp.1071-1077, 2012.

[2] Adebiyi, E.F., S.A. Fatumo, "Mathematical methods and their applications,” I ${ }^{\text {st }}$ Edition, Covenant University, Ota, Ogun State, Nigeria, 2006.

[3] Saberi-Nadjafi, J., M. Tamamgar, “The variational method: A highly promising method for solving system of integro-differential equations," Computers and Mathematics with Applications, vol. 56, pp.346-351. 2008.

[4] Matinfar, M., M. Ghanbari, "Solution of systems of integro-differential equations," Journal of Mathematics and Computer Science, vol. 1, pp.46-57, 2010.

[5] Mamadu, E.J., I.N. Njoseh, "On the convergence of variational iteration method for the numerical solution of nonlinear integro-differential equations," Transactions of the Nigerian Association of Mathematical Physics, vol. 2, pp. 65-70, 2016.

[6] Njoseh, I.N., E. J. Mamadu, "Modified Variation Iteration Homotopy Perturbation Method for the Approximate Solution of Burgers Equation," Transactions of Nigeria Association of Mathematical Physics, vol. 3, pp. $1-4$, 2017.

[7] J.H. He, "Variational iteration method for autonomous ordinary differential system,” Appl. Math. Comp.,vol. 114, no. 1, pp.114-123. 2000.

[8] J.H. He, "Variation Iteration Method: A kind of Nonlinear Analytical Technique: Some Example,” International Journal of Nonlinear Mechanics, vol. 34, no. 4, pp. 699-708, 1999.

[9] Abbvandy, S., E. Shivanian, “Application of the Variational Iteration Method for System of Nonlinear Volterra'sIntegro-differential equations," Mathematics and Computational Applications, vol. 14, no. 2, pp. 147-158, 2009.

[10] Kuma, D., M.K. Kadalbajoo, "Numerical treatment of singularly perturbed delay differential equations using B-spline collocation method on Shishkin mesh,” Journal of Numerical Analysis, Industrial and Applied Mathematics (JNAIAM), vol. 7, pp. 73 - 90, 2012.

[11] Njoseh, I.N., E.J. Mamadu, "Numerical solutions of generalized Nth order boundary value problems using power series approximation method,” Applied Mathematics, vol. 7, pp. 1215-1224, 2016. http://dx.doi.org/10.4236/am. 
2016.711107.

[12] M. Zennaro, “On the P-stability of one-step collocation for delay differential equations," InternationalSeries of Numerical Mathematics (ISNM), vol. 74, pp. 334-343, 1985.

[13] Ali, “A spectral method for pantograph-type delay differential equations and its convergence," Journal of Computational Mathematics, vol. 27, pp. 254-265, 2009.

[14] Mamadu, E.J., I. N. Njoseh, “On the convergences of variation iteration method for nonlinear integro-differential equations," Transactions of the Nigeria Association of Mathematics Physics, vol. 2, pp. 65-70, 2016.

[15] Mamadu, E.J., I. N. Njoseh, "Numerical solutions of Volterra equations using Galerkin method with certain orthogonal polynomials," Journal of Applied Mathematics and Physics, vol. 4, pp. 376-382, 2016.
[16] CheHaziqahCheHussin, Ahmed IzaniMd Ismail, AdemKilicman, Amirah Azmi, “Approximate Analytical Solutions of Nonlinear Korteweg-de Vries Equations Using Multistep Modified Reduced Differential Transform," Mathematics and Statistics, vol. 8, No. 2A, pp. 9-16, 2020. DOI: $10.13189 / \mathrm{ms} .2020 .081302$.

[17] Mahmoud M. Elborai, Khairia El-Said El-Nadi, “The parabolic Transform and Some Singular Integral Evolution Equations,” Mathematics and Statistics, vol. 8, No. 4, pp. 410-415, 2020. DOI: 10.13189/ms.2020.080406.

[18] W. X. Ma, J. Li, C. M. Khalique, “A study ofn lump solutions to generalized Hirota-Satsuma-Ito equation in (2+1)-dimensions," complexity, Vol. 2018, Article ID 9059858, 7 pages, 2018

[19] W. X. Ma, "A search for lump solutions to combined fourth-order nonlinear PDE in (2+1)-dimensions,” Journal of Applied Analysis and computation, Vol. 9, PP. 1319-1332, 2019. 